\title{
Prevalence of chromosomal rearrangements involving non-ETS genes in prostate cancer
}

\author{
MARTINA KLUTH $^{1 *}$, RAMI GALAL $^{1 *}$, ANTJE KROHN $^{1}$, JOACHIM WEISCHENFELDT $^{4}$, \\ CHRISTINA TSOURLAKIS ${ }^{1}$, LISA PAUSTIAN ${ }^{1}$, RAMIN AHRARY ${ }^{1}$, MALIK AHMED $^{1}$, \\ SEKANDER SCHERZAI $^{1}$, ANNE MEYER ${ }^{1}$, HÜSEYIN SIRMA ${ }^{1}$, JAN KORBEL ${ }^{4}$, GUIDO SAUTER ${ }^{1}$, \\ THORSTEN SCHLOMM $^{2,3}$, RONALD SIMON ${ }^{1}$ and SARAH MINNER ${ }^{1}$
}

${ }^{1}$ Institute of Pathology, ${ }^{2}$ Martini-Clinic, Prostate Cancer Center, and ${ }^{3}$ Department of Urology, Section for Translational

Prostate Cancer Research, University Medical Center Hamburg-Eppendorf; ${ }^{4}$ Genome Biology Unit, European Molecular Biology Laboratory (EMBL), D-69117 Heidelberg, Germany

Received November 25, 2014; Accepted December 30, 2014

DOI: 10.3892/ijo.2015.2855

\begin{abstract}
Prostate cancer is characterized by structural rearrangements, most frequently including translocations between androgen-dependent genes and members of the ETS family of transcription factor like TMPRSS2:ERG. In a recent whole genome sequencing study we identified 140 gene fusions that were unrelated to ETS genes in 11 prostate cancers. The aim of the present study was to estimate the prevalence of non-ETS gene fusions. We randomly selected 27 of these rearrangements and analyzed them by fluorescence in situ hybridization (FISH) in a tissue microarray format containing 500 prostate cancers. Using break-apart FISH probes for one fusion partner each, we found rearrangements of 13 (48\%) of the 27 analyzed genes in 300-400 analyzable cancers per gene. Recurrent breakage, often accompanied by partial deletion of the genes, was found for NCKAP $5, S H 3 B G R$ and TTC 3 in $3(0.8 \%)$ tumors each, as well as for ARNTL2 and ENOX1 in $2(0.5 \%)$ cancers each. One rearranged tumor sample was observed for each of VCL, ZNF578, IMMP2L, SLC16A12, PANK1, GPHN, LRPI and $Z H X 2$. Balanced rearrangements, indicating possible gene fusion, were found for ZNF578, SH3BGR, LPR12 and $Z H X 2$ in individual cancers only. The results of the present study confirm that rearrangements involving non-ETS genes occur in prostate cancer, but demonstrate that they are highly individual and typically non-recurrent.
\end{abstract}

\section{Introduction}

Prostate cancer is the most frequent malignancy in men. Although the majority of patients present with early stage

Correspondence to: Dr Sarah Minner, Institute of Pathology, University Medical Center Hamburg-Eppendorf, Hamburg, Germany

E-mail: s.minner@uke.de

*Contributed equally

Key words: prostate cancer, gene fusion, break-apart assay, deletion tumors that can be surgically treated in a curative manner, $20 \%$ of the tumors will progress to metastatic and hormone refractory disease, accounting for $>250.000$ deaths per year worldwide (1). Targeted therapies that would allow for an effective treatment after failure of androgen withdrawal therapy are lacking.

Recent whole genome sequencing studies have shown that the genomic landscape of prostate cancer differs markedly from that of other solid tumor types. Whereas, for example, breast or colon cancer is characterized by high-grade genetic instability and presence of a multitude of mutations, deletions, and amplifications including important therapy target genes such as HER2 and EGFR $(2,3)$, prostate cancers show only comparatively few mutations and almost completely lack amplifications (4-7). In contrast, prostate tumors are typically characterized by translocations, deletions, and gene fusions, the latter of which are recurrently involving androgen-responsive genes and transcription factors of the E-twenty six (ETS) family (8). The most frequent ETS-fusion is caused by interstitial deletion or translocation of a $3.7 \mathrm{Mb}$ genomic segment located between the TMPRSS2 serine protease and the $E R G$ transcription factor at chromosome 21q22. Approximately $50 \%$ of prostate cancers carry the TMPRSS2:ERG fusion, which brings $E R G$ under the control of the androgen responsive TMPRSS2 promoter and results in permanent expression of ERG (9). Accordingly, ETS-fusion proteins have been proposed as putative targets for future gene-specific therapies (10).

In a recent study, which was performed in the context of the International Cancer Genome Consortium (11) (ICGC) project on Early-Onset Prostate Cancer, we have carried out integrated genomic analyses, including whole-genome, transcriptome, and DNA methylome sequencing in 11 early onset prostate cancer (EO-PCA) patients and detected a total of 156 individual gene fusions, 140 of which were non-recurrent and unrelated to ETS genes (5). It could be possible that some of these rearrangements result in expressed fusion proteins that could serve as cancer-specific therapy targets, provided that these rearrangements occur at sufficient frequency to justify the efforts of drug development. Accordingly, the aim of the present study was to 
determine the prevalence of rearrangements of 27 genes by fluorescence in situ hybridization (FISH) analysis in 500 prostate cancer samples in a tissue microarray format.

\section{Materials and methods}

Tissues. A subset of our previously described prostate cancer prognosis tissue microarray (12) was used for the present study, including one TMA block containing one $0.6 \mathrm{~mm}$ punch each from formalin-fixed and paraffin-embedded tumor samples of 500 different patients undergoing surgery between 1992 and 2004 at the Department of Urology, University Medical Center Hamburg-Eppendorf. Presence of tumor cells in the tissue spots was confirmed in 478 tissue spots by $34 \beta \mathrm{BE} 12$ immunostaining in an adjacent TMA slide (13). The remaining 22 tissue spots were excluded from analysis. The pathological parameters of the TMA spots are described in Table I.

Fluorescence in situ hybridization (FISH). FISH was used to detect rearrangements of the 27 selected target genes. For all genes, dual color FISH break-apart probes were manufactured from Spectrum Orange/Spectrum Green labeled bacterial artificial chromosomes (BACs) corresponding to the $5^{\prime}$ and $3^{\prime}$ flanking regions of the individual genes. A list of the target genes, BAC clones, and labeling schemes is provided in Table II. For FISH analysis, freshly cut $4 \mu \mathrm{m}$ TMA sections were de-waxed and pre-treated using a commercial kit (paraffin pretreatment reagent kit; Abbott Molecular, Wiesbaden, Germany), followed by dehydration in 70,80 and $96 \%$ ethanol, air-drying and denaturation for $10 \mathrm{~min}$ at $72^{\circ} \mathrm{C}$ in $70 \%$ formamide-2X SSC solution. Hybridization was done overnight at $37^{\circ} \mathrm{C}$ in a humidified chamber; slides were then washed, counterstained with $0.2 \mu \mathrm{mol} / 1$ 4'-6-diamidino2-phenylindole in mounted in antifade solution.

Scoring of FISH. The stained slides were visually inspected under an epifluorescence microscope. A rearrangement was assumed if at least one split signal consisting of a separate orange and green signal was observed in $\geq 60 \%$ of the tumor cell nuclei (indicating balanced translocations) or if individual orange and green signals from the overlapping orange/green signal were lost (indicating deletions with breakpoint inside the gene or imbalanced translocations). Presence of only one overlapping orange/green signal in $>60 \%$ of tumor cells were considered heterozygous deletion. Tumors with complete lack of overlapping orange/green signals were regarded as homozygous deletions provided that FISH signals were present in adjacent normal cells.

\section{Results}

Rearrangements were detected for $13(48 \%)$ of the 27 tested genes. Recurrent breakage was found for NCKAP5, SH3BGR and TTC 3 in 3 tumors each, as well as for ARNTL2 and $E N O X 1$ in 2 cancers each. One rearranged tumor sample was observed for each of VCL, ZNF578, IMMP2L, SLC16A12, $P A N K 1, G P H N, L R P 1$ and $Z H X 2$. All but four rearrangement were unbalanced, i.e. either the $5^{\prime}$ or the $3^{\prime}$ part of the FISH probe was lost. For ZNF578, SH3BGR, LPR 12 and ZHX2 a split signal was found suggesting balanced translocation.
Table I. Composition of the prognosis TMA containing 500 prostate cancer specimens.

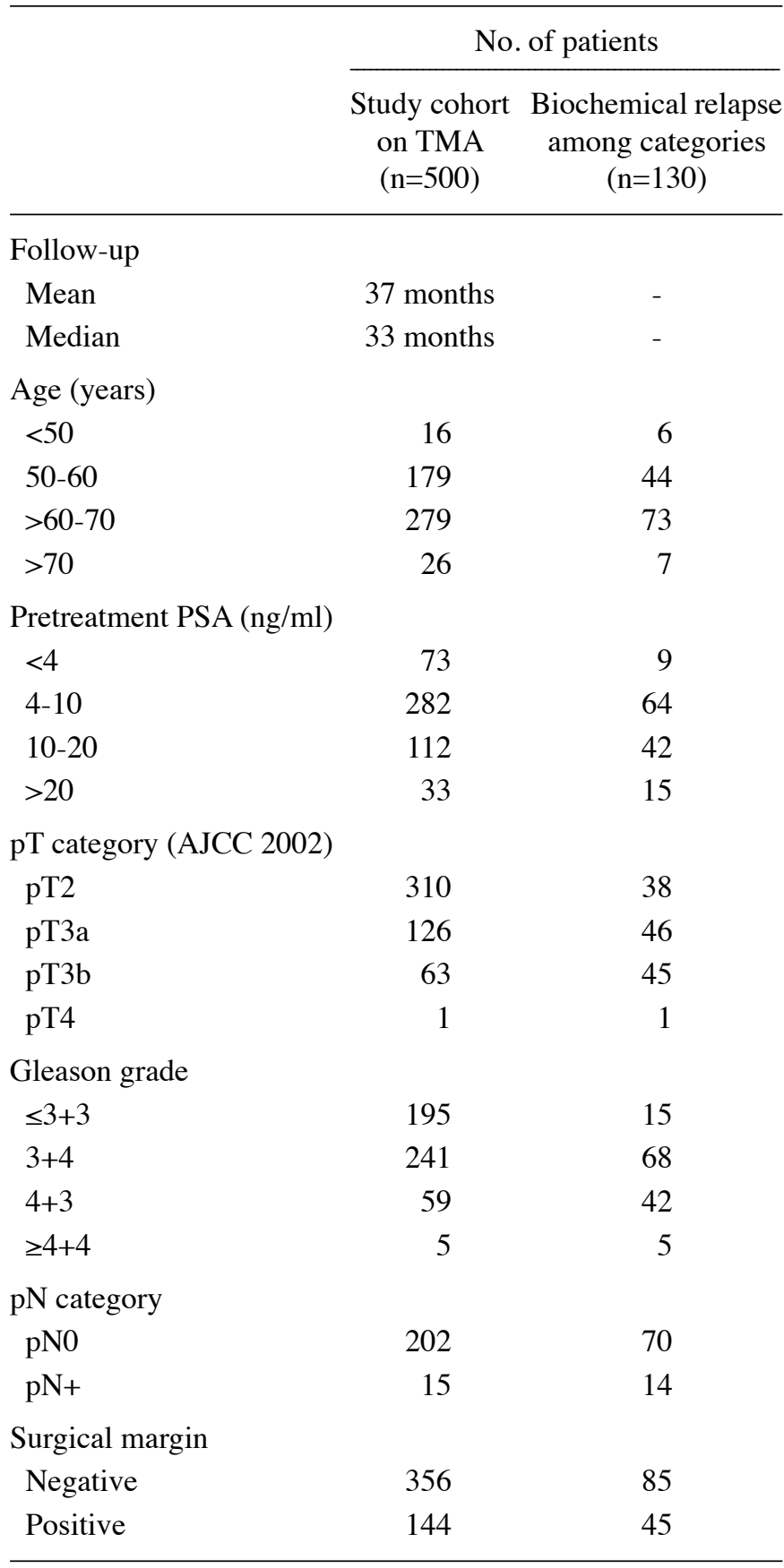

Numbers do not always add up to 500 in the different categories because of cases with missing data. AJCC, American Joint Committee on Cancer.

Deletions were markedly more frequent than translocations. The most frequently deleted genes were NCKAP5 (7.5\%), VCL (6.8\%), PANK1 (5.9\%), ARNTL2 (5.8\%), SLC16A12 (5.6\%), SH3BGR (3.0\%) and PCNXL2 (1.6\%). All detected deletions were heterozygous. No alternations were found for C1lorf41, MLLT4, ALDH7A1, EPN1, NR3C1, PACRG, LYRM4, DPF3, FAM154A and WDR67. The number of successfully analyzed samples per target gene, and the frequency and type of rearrangements and deletions for all analyzed genes is summarized in Table III. Representative FISH images are shown in Fig. 1. 
Table II. List of the genes that were analyzed for rearrangements using FISH break-apart probes.

\begin{tabular}{|c|c|c|c|c|c|}
\hline \multirow[b]{2}{*}{ Gene } & \multirow[b]{2}{*}{$\begin{array}{l}\text { Chromosomal } \\
\text { locus }\end{array}$} & \multicolumn{2}{|c|}{ FISH break apart probe composition } & \multicolumn{2}{|c|}{ Whole genome sequencing results ${ }^{a}$} \\
\hline & & 5' BAC(s) & $3^{\prime} \mathrm{BAC}(\mathrm{s})$ & $\begin{array}{l}\text { Rearrangement } \\
\text { type }\end{array}$ & Fusion partner genes \\
\hline$A L D H 7 A 1$ & $5 q 23.2$ & SO RP11-772E11 & SG RP11-517I3 & $\begin{array}{l}\text { Translocation } \\
\text { Translocation } \\
\text { Translocation }\end{array}$ & $\begin{array}{l}\text { ANKRD27:ALDH7A1 } \\
\text { ZNF480:ALDH7A1 } \\
\text { ELAVL1:ALDH7A1 }\end{array}$ \\
\hline$N R 3 C 1$ & $5 q 31.3$ & SG RP11-614D16 & SO RP11-738H11 & Translocation & NR3C1:HOXA9 \\
\hline SLC16A12 & $10 \mathrm{q} 23.31$ & SG RP11-788M08 & SO RP11-168010 & Translocation & SLC16A12:TESC \\
\hline FAM154A & $9 \mathrm{p} 22.1$ & SG RP11-151J10 & SO RP11-220B22 & $\begin{array}{l}\text { Translocation } \\
\text { Translocation }\end{array}$ & $\begin{array}{l}\text { FAM154A:IRAK3 } \\
\text { FAM154A:LRP1 }\end{array}$ \\
\hline PANK1 & $10 q 23.31$ & SG RP11-626K2 & SO RP11-705K1 & Translocation & CCNT1:PANK1 \\
\hline ARNTL2 & $12 \mathrm{p} 11.23$ & SG RP11-546C06 & SO RP11-529A16 & Translocation & $A R N T L 2$ \\
\hline ZNRF3 & $22 \mathrm{q} 12.1$ & $\begin{array}{l}\text { SO RP11-436H02, } \\
\text { SO RP11-493M06 }\end{array}$ & $\begin{array}{l}\text { SG RP11-664C16, } \\
\text { SG RP11-213L15 }\end{array}$ & Translocation & ZNRF3:FBXO16 \\
\hline$I M M P 2 L$ & $7 q 31.1$ & $\begin{array}{l}\text { SG RP11-365F8, } \\
\text { RP11-148C1 }\end{array}$ & $\begin{array}{l}\text { SO RP11-75O20, } \\
\text { RP11-154C19 }\end{array}$ & Translocation & $I M M P 2 L: L Y S T$ \\
\hline ENOX1 & $13 q 14.3$ & $\begin{array}{l}\text { SG RP11-75G24, } \\
\text { RP11-671N06 }\end{array}$ & $\begin{array}{l}\text { SO RP11-364B16, } \\
\text { RPRP11-64J21 }\end{array}$ & $\begin{array}{l}\text { Translocation } \\
\text { Translocation }\end{array}$ & $\begin{array}{l}\text { ENOX1:ANO2 } \\
\text { WWOX:ENOX1 }\end{array}$ \\
\hline LYRM4 & $5 \mathrm{p} 25.1$ & SO RP3-520B18 & SG RP11-284B11 & Translocation & $-: L Y R M 4$ \\
\hline CNOT10 & $3 \mathrm{p} 22.3$ & SO RP11-1005I1 & SG RP11-301L7 & Translocation & -:CNOT10 \\
\hline$H L C S$ & $21 \mathrm{q} 22.13$ & SG RP11-383L18 & SO RP11-169M12 & $\begin{array}{l}\text { Translocation } \\
\text { Inversion } \\
\text { Inversion } \\
\text { Translocation }\end{array}$ & $\begin{array}{l}\text { C1orf151:HLCS } \\
\text { HLCS:TTC3 } \\
\text { HLCS:ERG } \\
\text { TTC3:CCDC } 21\end{array}$ \\
\hline$T T C 3$ & $21 \mathrm{q} 22.13$ & SO RP11-674C12 & SG RP11-70N15 & $\begin{array}{l}\text { Inversion } \\
\text { Inversion }\end{array}$ & $\begin{array}{l}\text { TTC } 3: E R G \\
H L C S: T T C 3\end{array}$ \\
\hline PCNXL2 & $1 \mathrm{q} 42.2$ & SO RP11-740C10 & SG RP11-125H16 & $\begin{array}{l}\text { Translocation } \\
\text { Deletion } \\
\text { Deletion }\end{array}$ & $\begin{array}{l}\text { ENSG00000253819:PCNXL2 } \\
\text { DISC1:PCNXL2 } \\
\text { C11orf41:RAG1 }\end{array}$ \\
\hline C11orf41 & $11 \mathrm{p} 13$ & SG RP11-528E21 & SO RP11-60G13 & Deletion & C11orf41:OR51E2 \\
\hline MLLT4 & $6 q 27$ & SO RP11-351J23 & SG RP11-359F23 & Deletion & MLLT4:KIF25 \\
\hline$G P H N$ & $14 \mathrm{q} 23.3$ & $\begin{array}{l}\text { SG RP11-107B06, } \\
\text { SG RP11-100A18 }\end{array}$ & $\begin{array}{l}\text { SO RP11-205I6, } \\
\text { SO RP11-769005 }\end{array}$ & $\begin{array}{l}\text { Deletion } \\
\text { Deletion }\end{array}$ & $\begin{array}{l}\text { GPHN:RGS6 } \\
\text { GPHN:DPF3 }\end{array}$ \\
\hline$V C L$ & $10 \mathrm{q} 22.2$ & SG RP11-417O11 & SO RP11-178G16 & Deletion & VCL:ZNF503 \\
\hline$D P F 3$ & $14 \mathrm{q} 24.2$ & $\begin{array}{l}\text { SO RP5-1140N14, } \\
\text { SO RP11-326F24 }\end{array}$ & $\begin{array}{l}\text { SG RP11-437J15, } \\
\text { SG RP3-514A23 }\end{array}$ & $\begin{array}{l}\text { Deletion } \\
\text { Inversion } \\
\text { Inversion }\end{array}$ & $\begin{array}{l}G P H N: D P F 3 \\
R G S 6: D P F 3 \\
\text { ZNF578:EPN1 }\end{array}$ \\
\hline ZNF578 & $19 q 13.41$ & SO RP11-108N06 & SG RP11-207K02 & $\begin{array}{l}\text { Inversion } \\
\text { Inversion }\end{array}$ & $\begin{array}{l}\text { ANKRD27:ZNF578 } \\
\text { KDM4B:ZNF578 }\end{array}$ \\
\hline$S H 3 B G R$ & $21 \mathrm{q} 22.2$ & SG RP11-749C05 & SO RP11-165H11 & Inversion & SH3BGR:RIPK4 \\
\hline$L R P 12$ & $8 q 22.3$ & SO RP11-77K11 & SG RP11-437B02 & Inversion & LRP12:ENSG00000253350 \\
\hline$Z H X 2$ & $8 \mathrm{q} 24.13$ & SO RP11-94L20 & SG RP11-263A19 & Inversion & $-: Z H X 2$ \\
\hline WDR67 & $8 q 24.13$ & SG RP11-263A19 & SO RP11-54J08 & Inversion & ENSG00000254303:WDR67 \\
\hline EPN1 & $19 q 13.42$ & SO CTD-2537I9 & $\begin{array}{l}\text { SG CTD-2611012, } \\
\text { RP11-107J22 }\end{array}$ & Inversion & ZNF578:EPN1 \\
\hline NCKAP5 & $2 q 21.2$ & $\begin{array}{l}\text { SO RP11-736B01, } \\
\text { SO RP11-789J19 }\end{array}$ & $\begin{array}{l}\text { SG RP11-351L15, } \\
\text { SG RP11-393D01 }\end{array}$ & Inversion & NCKAP5:MGAT5 \\
\hline$P A C R G$ & $6 q 26$ & $\begin{array}{l}\text { SG RP11-57O22, } \\
\text { SG RP11-621H02 }\end{array}$ & $\begin{array}{l}\text { SO RP11-308E20, } \\
\text { SO RP3-495O10 }\end{array}$ & $\begin{array}{l}\text { Inversion } \\
\text { Duplication }\end{array}$ & $\begin{array}{l}\text { PACRG:LOC } 285796 \\
\text { IPCEF1:PACRG }\end{array}$ \\
\hline
\end{tabular}

SO, Spectrum Orange-labeled; SG, Spectrum Green-labeled. 'Data taken from Weischenfeldt et al (5). 
Table III. Prevalence and type of detected structural rearrangements.

\begin{tabular}{|c|c|c|c|c|c|c|}
\hline \multirow[b]{2}{*}{ Gene } & \multirow[b]{2}{*}{ Chromosomal locus } & \multicolumn{3}{|c|}{ Rearrangement } & \multicolumn{2}{|c|}{ Deletion } \\
\hline & & Analyzable & Unbalanced & Balanced & Analyzable & Deletion \\
\hline PCNXL2 & $1 \mathrm{q} 42.2$ & 436 & 0 & 0 & 436 & $7(1.6)$ \\
\hline NCKAP5 & $2 \mathrm{q} 21.2$ & 377 & $3(0.8)$ & 0 & 374 & $28(7.5)$ \\
\hline CNOT10 & $3 \mathrm{p} 22.3$ & 382 & 0 & 0 & 382 & $4(1.0)$ \\
\hline$I M M P 2 L$ & 7q31.1 & 320 & $1(0.3)$ & 0 & 320 & $1(0.3)$ \\
\hline LRP12 & $8 \mathrm{q} 22.3$ & 321 & 0 & $1(0.3)$ & 321 & 0 \\
\hline$Z H X 2$ & $8 \mathrm{q} 24.13$ & 389 & 0 & $1(0.3)$ & 389 & 0 \\
\hline$V C L$ & $10 \mathrm{q} 22.2$ & 338 & $1(0.3)$ & 0 & 176 & $12(6.8)$ \\
\hline SLC16A12 & $10 \mathrm{q} 23.31$ & 363 & $1(0.3)$ & 0 & 250 & $14(5.6)$ \\
\hline PANKI & $10 q 23.31$ & 355 & $1(0.3)$ & 0 & 188 & $11(5.9)$ \\
\hline ARNTL2 & $12 \mathrm{p} 11.23$ & 316 & $2(0.6)$ & 0 & 171 & $10(5.8)$ \\
\hline ENOXI & 13q14.3 & 435 & $2(0.5)$ & 0 & 435 & 0 \\
\hline$G P H N$ & $14 \mathrm{q} 23.3$ & 406 & $1(0.2)$ & 0 & 406 & 0 \\
\hline ZNF578 & $19 q 13.41$ & 393 & 0 & $1(0.3)$ & 393 & 0 \\
\hline$H L C S$ & $21 \mathrm{q} 22.13$ & 360 & 0 & 0 & 360 & $2(0.6)$ \\
\hline TTC3 & $21 \mathrm{q} 22.13$ & 385 & $3(0.8)$ & 0 & 385 & 0 \\
\hline$S H 3 B G R$ & $21 \mathrm{q} 22.2$ & 368 & $2(0.5)$ & $1(0.3)$ & 368 & $11(3.0)$ \\
\hline ZNRF3 & $22 \mathrm{q} 12.1$ & 273 & 0 & 0 & 273 & $4(1.5)$ \\
\hline
\end{tabular}

\section{Discussion}

The results of the present study demonstrate that most chromosomal rearrangement, including balanced translocations and partial deletions characterized by intragenic breaks, represent very rare events in prostate cancer. The prevalence of breakage events affecting the 27 analyzed genes in this study was usually below $1 \%$.

Based on our data, obtained in a cohort of over 500 tumors, it is not surprising that whole genome sequencing studies on prostate cancer found only few recurrent rearrangements (except TMPRSS2:ERG) in a total of 18 cancers $(4,5)$. Although $>250$ individual non-ETS gene fusion events (resulting from translocations, inversions and duplications) were identified in these two studies in total, only 16 non-ETS genes in the study by Berger et al (4) and 1 gene in the study by Weischenfeld et al (5) were recurrently hit by structural rearrangements, however, in each case there was a different fusion partner. Only ETS-fusions were highly recurrent in these studies, with 4/7 tumors (4) and 8/11 tumors (5) carrying the TMPRSS2:ERG fusion.

Little is known about the prevalence of individual gene rearrangements (except TMPRRS2:ERG) in prostate cancer. Two studies performed by Reid et al (14) and us analyzed breakage of the PTEN tumor suppressor, and reported 7\% (13/187) (14) and $3 \%(162 / 5,404)(5)$ of PTEN breakage, which was typically ( 3 out of 4 affected cases) associated with deletions of the second PTEN allele. In addition, we have previously studied breakage of the 3 p13 tumor suppressor FOXP1 (15) and found $1.2 \%$ of rearrangements. These data suggest that rearrangements are infrequent even for genes with a key role including PTEN. The $0.2-1 \%$ of rearrangements found for half of the genes analyzed in the present study fit well to these numbers.
The selection of the 27 genes analyzed in this study was based on the findings of our International Cancer Genome (ICGC) project, where we employed the paired end deep sequencing strategy (16) to specifically identify gene breakages, translocations and gene fusions. In the present study we found a total of 140 non-ETS gene rearrangements. For the present study, we randomly selected genes that were potentially involved in non-ETS fusions between protein-coding genes or gene inactivation by translocation or gene breakage (5). Such genes are candidates for a dual tumor relevant function, including a putative tumor suppressor function based on inactivation by gene breakage, as well as a putative oncogenic in case of expressed fusion genes.

In this study, deletion of the analyzed region was more frequent than rearrangement. This fits well with the known relevance of many of the analyzed genes, which were located at chromosomal regions that are frequently deleted on prostate cancer, including for example PANK1, VCL and SLC16A12 (10q22-q23, deleted in 20-30\%) (17-19), NCKAP5 (2q21, deleted in 10-30\%) (17-19), or ARNTL2 (12p11-p12, deleted in $15-60 \%)(17,19)$, explaining the markedly higher frequency of deletions as compared to rearrangements. The deletion frequencies observed in the present study were markedly lower than in these studies, which can be explained by the fact that we did not use a deletion-specific FISH assay including a combination of a locus-specific and a centromere reference probe. With the break-apart probe used in this study, we only called absolute deletions showing unequivocal loss of one red-green signal pair but missed relative deletions, which frequently occur in aneuploid cancers.

Several of the genes analyzed in this study, including NCKAP5:MGAT5, C11orf41:RAG1, SH3BGR:RIPK4, FAM154A:IRAK3 and CCNT1:PANK1, were involved in 
A

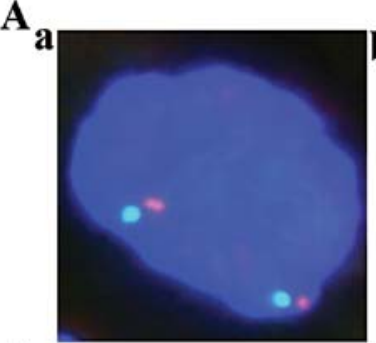

C

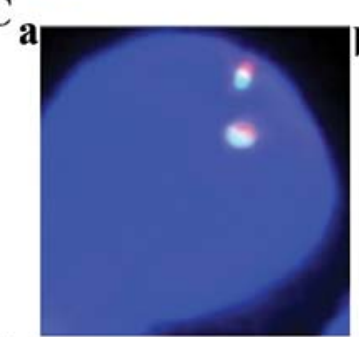

$\mathbf{E}$

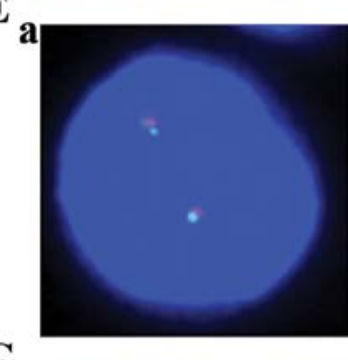

G

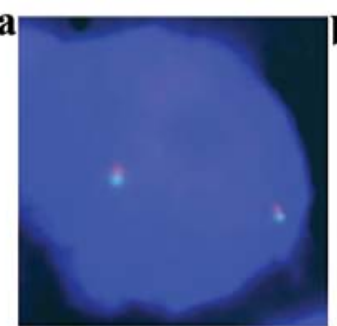

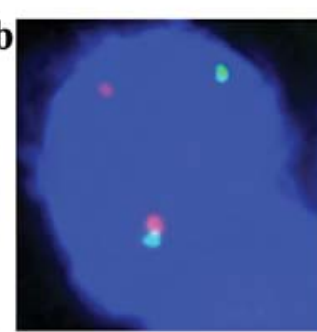
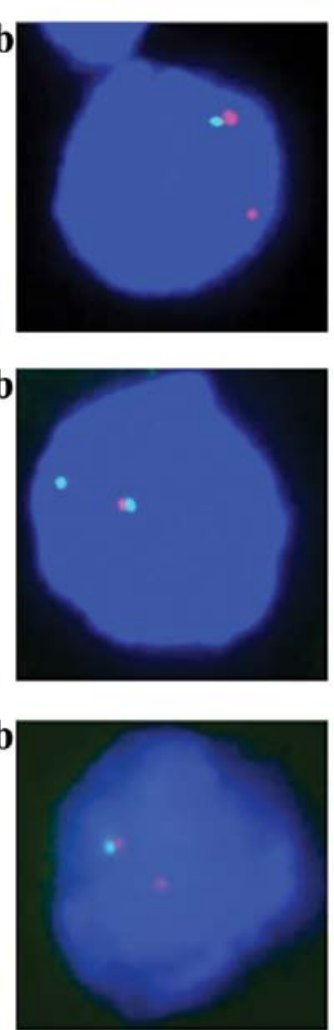

B


D
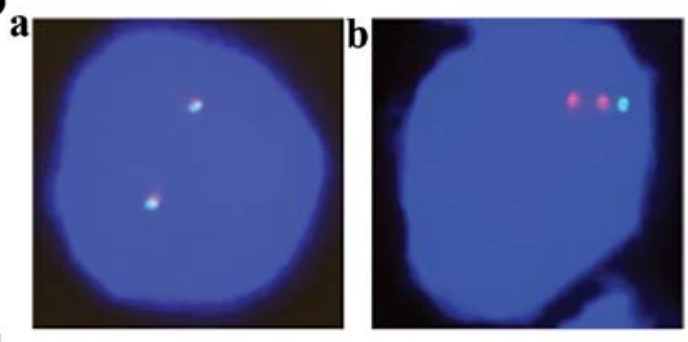

F
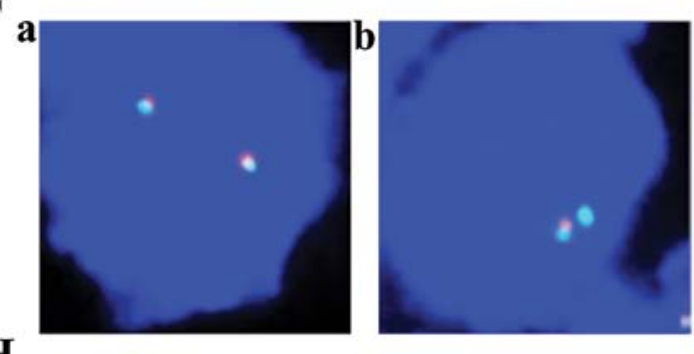

.
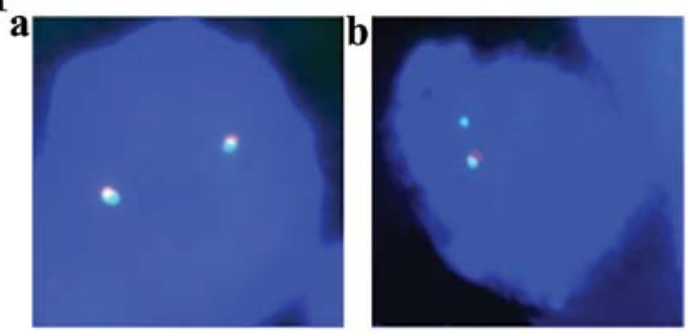

Figure 1. Examples of FISH findings using the break-apart probes. (Aa-Ha) Intact gene locus of LRP12, ZHX2, ARNTL2, ENOX1, IMMP2L, GPHN, ZNF578 and NCKAP5 with two adjacent green and orange FISG signals corresponding to the $3^{\prime}$ and $5^{\prime}$ flanking regions of these genes. (Ab) Breakage of one $L R P 12$ allele as indicated by a split signal (separate red and green signals) while the second allele is still intact. (Bb) Breakage of two $Z H X 2$ alleles as indicated by two separate red and green signals. (Cb-Hb) Breakage of one gene allele as indicated by a loss of one red signal of $I M M P 2 L(\mathrm{~Eb}), G P H N(\mathrm{Fb})$ and $N C K A P 5(\mathrm{Hb})$ or by a loss of one green signal of ARNTL2 (Cb), ENOX1 (Db) and ZNF578 (Gb).

gene fusions leading to overexpression of the fusion partner according to our previous study (5). Such fusion genes may represent suitable targets for new gene specific therapies, since they are specific for the cancer cells. However, the vast majority of gene breakages detected in this study were unbalanced, with loss of either the $3^{\prime}$ or the $5^{\prime}$ fraction of the gene, suggesting a partial deletion of these genes. Only 4 genes, ZNF587, SH3BGR, LRP12 and ZHX2, showed balanced rearrangements that might have led to gene fusions. These findings suggest that intragenic breaks may in most cases indicate a deletion break point located inside a coding gene, while formation of a specific rearrangement with a possible functional fusion gene seems to be a comparatively rare event.

We manufactured break-apart probe assays to detect rearrangements of the 27 candidate genes in a tissue microarray format. The use of our tissue microarray format in combination with FISH enables a fast and cheap analysis of gene rearrangements to detect common recurrent gene changes. Break-apart assays are capable of detecting all types of rearrangements of a probed gene, including translocation, (partial) deletion and inversion, and are thus optimally suited to estimate the prevalence of rearrangements for a given gene. We selected a cut-off level of $\geq 60 \%$ affected tumor cell nuclei for the detection of rearrangements in order to avoid false-positive findings due to truncated cell nuclei in $4 \mu \mathrm{m}$ tissue sections. This cut-off was based on our previous studies analyzing breakage of $E R G(20)$ and PTEN $(5,17)$. Using this threshold we found a high $(>95 \%)$ correlation between $E R G$ breakage by FISH and ERG expression be immunohistochemistry (20), supporting the validity of our approach to screen for recurrent gene rearrangements.

In summary, the present study shows that a multitude of genes can be affected by chromosomal rearrangements in prostate cancer, but the frequency of specific rearrangements is typically in the range of $1 \%$ or less. In most cases, these rearrangements will result in gross deletions inactivating the affected gene. True translocations, potentially resulting in fusion genes, are comparatively rare. 


\section{References}

1. Jemal A, Siegel R, Xu J and Ward E: Cancer statistics, 2010. CA Cancer J Clin 60: 277-300, 2010

2. Sjoblom T, Jones S, Wood LD, et al: The consensus coding sequences of human breast and colorectal cancers. Science 314 268-274, 2006.

3. Wood LD, Parsons DW, Jones S, et al: The genomic landscapes of human breast and colorectal cancers. Science 318: 1108-1113, 2007.

4. Berger MF, Lawrence MS, Demichelis F, et al: The genomic complexity of primary human prostate cancer. Nature 470 214-220, 2011.

5. Weischenfeldt J, Simon R, Feuerbach L, et al: Integrative genomic analyses reveal androgen-driven somatic alteration landscape in early-onset prostate cancer. Cancer Cell 23: 159-170, 2013.

6. Barbieri CE, Baca SC, Lawrence MS, et al: Exome sequencing identifies recurrent SPOP, FOXA1 and MED12 mutations in prostate cancer. Nat Genet 44: 685-689, 2012.

7. Grasso CS, Wu YM, Robinson DR, et al: The mutational landscape of lethal castration-resistant prostate cancer. Nature 487: 239-243, 2012.

8. Tomlins SA, Rhodes DR, Perner S, et al: Recurrent fusion of TMPRSS2 and ETS transcription factor genes in prostate cancer. Science 310: 644-648, 2005.

9. Tomlins SA, Bjartell A, Chinnaiyan AM, et al: ETS gene fusions in prostate cancer: from discovery to daily clinical practice. Eur Urol 56: 275-286, 2009.

10. Shao L, Tekedereli I, Wang J, et al: Highly specific targeting of the TMPRSS2/ERG fusion gene using liposomal nanovectors. Clin Cancer Res 18: 6648-6657, 2012.
11. Hudson TJ, Anderson W, Artez A, et al: International network of cancer genome projects. Nature 464: 993-998, 2010.

12. Schlomm T, Chun F and Erbersdobler A: From gene to clinic: TMA-based clinical validation of molecular markers in prostate cancer. Methods Mol Biol 664: 177-189, 2010.

13. Minner S, Jessen B, Stiedenroth L, et al: Low level HER2 overexpression is associated with rapid tumor cell proliferation and poor prognosis in prostate cancer. Clin Cancer Res 16: 1553-1560, 2010.

14. Reid AH, Attard G, Brewer D, et al: Novel, gross chromosomal alterations involving PTEN cooperate with allelic loss in prostate cancer. Mod Pathol 25: 902-910, 2012.

15. Krohn A, Seidel A, Burkhardt L, et al: Recurrent deletion of 3 p13 targets multiple tumor suppressor genes and defines a distinct subgroup of aggressive ERG fusion positive prostate cancers. J Pathol 231: 130-141, 2013.

16. Korbel JO, Urban AE, Affourtit JP, et al: Paired-end mapping reveals extensive structural variation in the human genome. Science 318: 420-426, 2007.

17. Krohn A, Diedler T, Burkhardt L, et al: Genomic deletion of PTEN is associated with tumor progression and early PSA recurrence in ERG fusion-positive and fusion-negative prostate cancer. Am J Pathol 181: 401-412, 2012.

18. Sun J, Liu W, Adams TS, et al: DNA copy number alterations in prostate cancers: a combined analysis of published CGH studies. Prostate 67: 692-700, 2007.

19. Taylor BS, Schultz N, Hieronymus H, et al: Integrative genomic profiling of human prostate cancer. Cancer Cell 18: 11-22, 2010.

20. Minner S, Enodien M, Sirma H, et al: ERG status is unrelated to PSA recurrence in radically operated prostate cancer in the absence of antihormonal therapy. Clin Cancer Res 17: 5878-5888, 2011. 